\title{
Schmerzmanagement bei fortgeschrittener Demenz
}

\author{
Von der Neurobiologie zur praktischen Anwendung
}

Typischerweise liegen bei geriatrischen Patienten mehrere Erkrankungen gleichzeitig vor, die sich wechselseitig in ihrem Verlauf, aber auch in ihrer symptomatischen Auswirkung, beeinflussen. Dies gilt besonders für das Zusammentreffen chronischer Schmerzen mit einer Demenz.

In der Gruppe der über 50-Jährigen leiden 43 Prozent unter Dauerschmerz. Etwa drei Viertel der über 74-jährigen Personen berichten über Schmerzen, davon ein Drittel über schwere Dauerschmerzen. Bei Heimbewohnern sind chronische Schmerzen in bis zu 80 Prozent der Fälle nachweisbar, ein Drittel davon leidet an Dauerschmerzen. Die Ursachen von chronischen Schmerzen im Alter sind vor allem degenerative Wirbelsäulen- und Gelenkserkrankungen, Krebsschmerzen, Schmerzen bei Osteoporose, Gürtelrose oder rheumatische Schmerzen, Nervenschmerzen infolge eines Diabetes mellitus oder Schmerzen infolge von Stürzen und Frakturen.

Demente Patienten mit chronischen oder starken Schmerzen können bereits in frühen Erkrankungsphasen aufgrund der eingeschränkten verbalen Kommunikationsfähigkeit ihre Schmerzen nicht entsprechend mitteilen. Klassische SchmerzAssessment-Instrumente wie die VAS (Visuelle Analog-Skala), numerische Skalen oder Smiley-Skalen können zumeist nicht mehr sinnvoll eingesetzt werden. In der Folge kommt es dazu, dass Ärzte und Pflegepersonen das Problem nur unzureichend wahrnehmen und unterschätzen, eine adäquate Schmerztherapie wird in einer Vielzahl der Fälle nicht durchgeführt.

\section{Demenz-bedingte Veränderungen}

Aktuelle Untersuchungen mittels funktioneller Imaging-Methoden (PET bzw. f-MRT) legen den Schluss nahe, dass Alzheimer-Patienten nicht weniger Schmerz empfinden als nicht-demente Patienten. Man geht davon aus, dass die Schmerzwahrnehmung aufgrund der progredienten neuropathologischen Veränderungen infolge der Demenzerkrankung vor allem jene Bereiche des zentralen, schmerzverarbeitenden Systems (Pain-Matrix) im Gehirn betreffen, die primär für die emotional-af- fektive Komponente des Schmerzerlebens sowie die kognitiv evaluative Integration des Schmerzerlebens verantwortlich sind. Die sensorisch diskriminativen Schmerzaspekte bleiben hingegen bis in die späten Stadien der Demenz relativ unbeeinflusst.

Aufgrund der demenzbedingten Veränderungen des schmerzverarbeitenden Systems muss man heute davon ausgehen, dass es bei Demenzerkrankung zu einer veränderten Schmerzwahrnehmung mit den Folgen einer verschleierten Schmerzsymptomatik kommt. Man nimmt an, dass Schmerzen bei Dementen sogar zu wesentlich stärkeren Reaktionen führen, wie zum Beispiel Unruhe, Umtriebigkeit, lautes Schreien oder Aggressivität. Motorische Fluchtreflexe können schlechter unterdrückt und kontrolliert werden, die Integration der Schmerzwahrnehmung gelingt schlechter, was sich etwa in einer allgemein gesteigerten psychomotorischen Unruhe spiegelt. Somit wird vorstellbar, dass Schmerzen aufgrund ihres interruptiven Charakters Aufmerksamkeit und vorhandene kognitive Kapazitäten in einem besonderen Ausmaß binden und dadurch eine besondere Bedrohung darstellen.

Eine neue Erkenntnis mit noch nicht restlos geklärter Bedeutung für die klinische Praxis sind Hinweise aus experimentellen Studien, dass Demenzpatienten nur mehr eingeschränkt in der Lage sind, einen Placeboeffekt - der sich ja auf Grundlage eines intakten präfrontalen Kortex beziehungsweise deszendierenden SchmerzInhibitionssystems erst ausbilden kann zu generieren. Es scheint möglich, dass Demenzpatienten dadurch nur eingeschränkt von der Gabe eines Analgetikums profitieren, da der Placeboeffekt ja bekanntlich bis zu 50 Prozent der gesamten Medikamentenwirkung ausmacht.

\section{Klinisches Schmerzmanagement}

Als Konsequenz für das klinische Schmerzmanagement bei Demenz ist es daher notwendig, zuerst die Frage nach einer vorliegenden kognitiven Einschränkung, also dem Vorliegen einer Demenz, zu klären. Hier stehen als Screening-Instrumente vor allem der MMSE bzw. der Uhrentest, aber auch der Drei-Wörter-Uhrentest als pra- xistaugliche Instrumente in der Ordination zur Verfügung. In der Folge sollte eine fachärztliche Demenzdiagnostik erfolgen und eine Therapie eingeleitet werden. Mit modernen Screening- und Diagnosemethoden ist es heute ebenfalls möglich, auch bei fortgeschritten dementen Patienten, die ihre Schmerzen nicht adäquat mitteilen können, diese zuverlässig zu erfassen. Dafür werden beispielsweise die BESD-Skala (Beurteilung von Schmerz bei Demenz) oder der DOLOPLUS2-Screeningbogen eingesetzt. Diese Beobachtungsverfahren orientieren sich an verschiedenen Zeichen auf der Verhaltensebene (motorische Symptome, verbale Äußerungen, Reaktion auf Trost, Atemmuster, Grimassieren etc.). Wesentlich ist, dass diese Screeningtests nach

\section{Demente Patienten können ihre Schmerzen nicht entsprechend mitteilen.}

einer entsprechenden Schulung prinzipiell nicht nur von medizinischem Personal, sondern auch von Pflegepersonen oder Angehörigen angewandt werden können.

Für die Therapie von Schmerzen bei Demenz gilt es zu beachten, dass aufgrund der mangelnden Compliance eine PCA (Patienten-gesteuerte Analgesie) oder „on demand" (bedarfsorientierte) Schmerztherapie nicht zielführend ist, sondern nach einem fixen, vorgegebenen Schema zu erfolgen hat. Für die Behandlung chronischer schwerer Schmerzzustände kann entsprechend dem WHO-Schema auch der Einsatz von (transdermalen) Opioiden sinnvoll sein. Daneben ist es ebenfalls notwendig, eine stadienspezifische Therapie der Demenzerkrankung durchzuführen. Auch hier stehen heute mit, auch transdermal verabreichbaren, Acetylcholinesterasehemmern und Memantin wirksame und sichere Therapieoptionen zur Verfügung, die über eine Verbesserung der Kognition einen günstigen Einfluss auf die Schmerzverarbeitung und -wahrnehmung leisten können.

\section{Korrepondenz}

Prim. Dr. Andreas Winkler, MSc

Ärztlicher Direktor der Klinik Pirawarth und Vorstand der Abteilung für Neurologische Rehabilitation Tel.: 02574 29160-0, E-Mail: info@klinik-pirawarth.at 